\title{
UNCERTAIN BUT ABLE: ENTREPRENEURIAL SELF-EFFICACY AND NOVICES' USE OF EXPERT DECISION-LOGIC UNDER UNCERTAINTY
}

\author{
Yuval Engel* \\ VU University Amsterdam \\ Nicoletta Dimitrova \\ VU University Amsterdam \\ Svetlana Khapova \\ VU University Amsterdam \\ Tom Elfring \\ VU University Amsterdam
}

\section{ACKNOWLEDGEMENTS}

We are grateful to Shabie Shafei and Martine Slütter for all their help and support during the data collection for this study. We thank Alexander McKelvie and participants of the BCERC 2014 as well as the 2014 SMS special conference in Copenhagen for their developmental comments on earlier versions of this paper. In addition, we gratefully acknowledge the highly valuable suggestions from editor Dimo Dimov on prior drafts. The study was supported by a grant from the Netherlands Organisation for Scientific Research (NWO).

\footnotetext{
* Corresponding author:

Yuval Engel, Faculty of Economics and Business Administration, VU University Amsterdam, De Boelelaan 1105, 1081 HV Amsterdam, The Netherlands, Tel: +31 20598 3550, e-mail: y.engel@ vu.nl

(C) Copyright 2014, Yuval Engel. All rights reserved. This paper is for the reader's personal use only. This paper may not be quoted, reproduced, distributed, transmitted or retransmitted, performed, displayed, downloaded, or adapted in any medium for any purpose, including, without limitations, teaching purposes, without the Author's express written permission. Permission requests should be directed to y.engel@vu.nl.
} 


\title{
Uncertain but able: Entrepreneurial self-efficacy and novices' use of expert decision-logic under uncertainty
}

\begin{abstract}
Entrepreneurs' initial strategy choices are made in the face of inherently uncertain and fundamentally unpredictable futures. Yet, unlike experts, novice entrepreneurs still tend to rely on predictions and forecasts as they move their ideas through the venture creation process. This study examines the role of entrepreneurial self-efficacy (ESE) and situational framing in mitigating the seemingly negative consequences of an "experience deficit" and promoting the use of effectuation - a non-predictive logic associated with entrepreneurial expertise. The results of a randomized experiment show that, in contrast to a control group and a low ESE group, novices who experienced an increase in ESE were more likely to use effectuation under uncertainty. This relationship was mediated by the framing of the situation as an opportunity.
\end{abstract}

Keywords: effectuation; entrepreneurial self-efficacy; opportunity; uncertainty; experiment

\section{Introduction}

Entrepreneurs' initial decisions are made in the face of uncertainty, which renders predictive decision-strategies as largely inadequate (Alvarez and Barney, 2007; Brinckmann et al., 2010; Miller, 2007; Wiltbank et al., 2006). Against this backdrop, 'effectuation' - a non-predictive logic used by expert entrepreneurs - is positioned as a viable alternative (Sarasvathy, 2001). Effectual logic does not require foresight but is rather concerned with shaping only these elements of the future that are currently within one's direct control (Sarasvathy, 2001; Wiltbank et al., 2006). By converging on a specific set of heuristics effectuation research is now starting to flesh out the logic's implications for the entrepreneurial process (Sarasvathy, 2008; Read et al., 2009). Yet, despite the rapidly growing volume of scholarship devoted to effectuation (see Perry et al., 2011 for a recent review), research on its antecedents remains limited in scope.

Prior work found that, unlike novices, highly experienced entrepreneurs unequivocally rely on effectuation (Dew et al., 2009; Sarasvathy, 2001). The central assumption in interpreting these findings is that the development of expertise in starting new ventures leads to important changes in how individuals think through decisions (Dew et al., 2009). However, this assumption was never directly tested and research to date falls short in rejecting alternatives to experience-based explanations (Baron, 2009). In light of recent studies documenting the use of effectuation by complete novices (e.g., Brettel et al., 2012; Chandler et al., 2011), it is evident that theory still does not adequately explain why entrepreneurs, confronted with identical conditions, vary in their reliance on effectuation?

We offer a new perspective to explain this question. Rather than making attributions to under-specified constructs such as experience or expertise we purposively attend to 
the other side of the "experience coin" - that is, the study of inexperienced entrepreneurs (e.g., Haynie et al., 2010b). This setting represents a highly relevant, if understudied, empirical context and allows us to complement prior research while avoiding the conflation of other explanatory constructs with entrepreneurial experience. Using these boundary conditions as a starting point, we focus our investigation on the role of entrepreneurial self-efficacy (ESE) - a single construct that has not only been shown to impact entrepreneurial decision-making more generally (Chen et al., 1998; Hmieleski and Baron, 2008) but was also overlooked in prior research about effectuation.

\section{ESE and effectuation: Uncertainty as opportunity}

Rooted in social cognitive theory (Bandura, 1997), ESE reflects the strengths of one's confidence in the ability to perform entrepreneurial-tasks (Chen et al., 1998). Importantly, even when lacking any prior experience, individuals may be highly confident (Camerer and Lovallo, 1999; Townsend et al., 2010) and entrepreneurial decisions are often attributed to this (over)confidence in ability (Hayward et al., 2006; Koellinger et al., 2007; Wu and Knott, 2006). These features position ESE as an important antecedent of decision-making in general, but also as a common factor that may be shared by experts and novices alike. Moreover, ESE stimulates heuristic thinking and propagates the belief in the ability to directly shape the environment (Wood and Bandura, 1989; Busenitz and Barney, 1997)., Hence, to the extent that effectual logic operates by playing down predictive information but in the same time utilizing proactiveness, agency, and control, ESE is clearly a conceivable predictor (Sarasvathy and Dew, 2008; Sarasvathy, 2008).

Into that context, we piece together Dutton and Jackson's (1987) work on situational framing to better understand how the same uncertain situation may be interpreted through different lenses, thereby evoking different strategic responses. When approaching decision-problems entrepreneurs convert the information they face into a meaningful picture through the imposition of simplified categories such as "opportunity" and "threat" (Dutton and Jackson, 1987). Unlike threats, opportunities carry positive meaning, and are associated with feelings of control (Jackson and Dutton, 1988). These salient characteristics of the opportunity frame provide the theoretical justification linking it to both ESE and effectuation (Gartner et al., 2008; Krueger and Dickson, 1994; Wiltbank et al., 2006). Simply put, when individuals feel confident in their entrepreneurial ability they are more likely to feel positive about their ability to control an uncertain environment, frame it as an opportunity, and use effectual logic. Figure 1 summarizes our hypotheses.

INSERT FIGURE 1 ABOUT HERE

\section{Method}

3.1. Participants and design 
The study sample consisted of 93 business and economics students at a Dutch university (57 females and 36 males; $M$ age $=23.80, S D_{\text {age }}=1.61$ ) who indicated having no prior entrepreneurial experience. We used a three-group betweenparticipant experimental design (Low ESE vs. High ESE vs. Control)

\subsection{Procedure and materials}

First, we checked whether randomization was successful by collecting data on demographics, ESE, meta-cognitive ability, and risk-propensity. Next, as a manipulation, participants received (bogus) feedback about their entrepreneurial ability, which was said to have been derived from the scoring of the answers given so far (see Gatewood et al., 2003). Participants in the control condition received no feedback. As a manipulation check, all participants were asked to answer questions related to their confidence in their entrepreneurial abilities (ESE). Participants then read a realistic venture scenario, specifically designed to elicit perceptions of high state uncertainty (see full description below). Participants were instructed to assume the role of the entrepreneur and answer questions about (1) their perception of state uncertainty in this situation, (2) their perception of the situation as an opportunity and threat, and (3) their decision-making logic in completing a series of venturing tasks. Finally, participants were debriefed.

\subsection{Measures $^{1}$}

Venture scenario, state uncertainty and effectual logic. Effectual logic was measured with 14 items by Witbank and colleagues (2009). Six items measured the reliance on predictive logic $(\alpha=.87)$ and eight items measured the reliance on non-predictive logic $(\alpha=.91)$. For our analysis and consistent with Murnieks et al., (2011) we operationalized the variable "effectual logic" as the distance between respondents" non-predictive and predictive scores, where larger positive scores denote stronger preferences for effectuation (negative for causation). This choice is supported conceptually (e.g., Sarasvathy, 2008) and empirically, as evident by the high negative correlation between the non-predictive and predictive scales $(r=-.67)$.

We developed a new high level state uncertainty venture scenario by using McKelvie et al's (2011, p. 288) specific operationalizations of state uncertainty. Respondents perception of state uncertainty was measured with a 4-item scale by Ashill and Jobber $(2010 ; \alpha=.88) .^{2}$

Opportunity framing. We operationalized opportunity framing by combining two items from Mohammed and Billings (2002) with higher scores reflecting an opportunity framing and lower scores reflecting threat framing $(\alpha=.77)$.

\footnotetext{
${ }^{1}$ Unless otherwise indicated, all measures were anchored on 5-point Likert scales ranging from 1 (strongly disagree) to 5 (strongly agree).

2 The venturing scenario and the decision-making questionnaire are provided in Appendix A.
} 
Manipulation checks. As a manipulation check we used a self-developed five-item scale, consistent with the ESE factors from Forbes (2005) - marketing, innovation, management, risk-taking, and finance - and with our experimental manipulation ( $\alpha$ $=.93)$.

Control variables. Meta-cognitive experience and meta-cognitive knowledge were measured using Haynie and Shepherds' (2009) scales. Risk propensity was measured with Mullins and Forlani’s (2005) scale.

\section{Results}

\subsection{Preliminary analysis}

The means and standard deviations per group, as well as all correlations can be found in Table 1. A multivariate analysis of variance (using Pilai's trace), with condition as the independent variable and all control variables as dependent variables, showed no significant multivariate effects, $V=.19, F(12,172)=1.56, p=.107$, but there was a univariate effect on age, $F(2,90)=3.29, p=.042$. We can therefore conclude that randomization into the experimental conditions was successful, with the exception of age.

The manipulation was successful in inducing high ESE. ANOVA showed a significant effect of condition on the manipulation check, $F(2,90)=38.95, p<.001$. High ESE group participants were significantly more certain of their entrepreneurial abilities than Low ESE and control group participants (for means see Table 1). No differences were found between the low ESE group and the control group, rendering the negative induction of ESE as unsuccessful. Additionally, as intended, participants across all conditions perceived the environment in the venture scenario as highly uncertain $(M=4.15), F(2,90)=0.004, p=.996$.

\section{INSERT TABLE 1 ABOUT HERE}

\subsection{Hypotheses testing}

ESE and effectual logic. ANOVA showed a significant effect of condition on effectual logic, $F(2,87)=36.72, p<$. 001. Simple contrast analyses showed that participants with high ESE were more likely to apply effectual logic than participants with low ESE, $t(87)=-6.81, p<.001$ or participants in the control group, $t(87)=-$ $7.91, p<.001$. No differences in preference for effectual logic were found between the low ESE and the control group, $t(87)=1.10, p=.276$ (see Figure 3 ).

\section{INSERT FIGURE 2 ABOUT HERE}

Opportunity framing as a mediator. We applied Hayes and Preacher's (2013) MEDIATE macro for SPSS, which allows for a multicategorical independent variable 
(5000 bootstrap resamples). The high ESE group was chosen as reference because participants' responses were consistently different from the responses of the low ESE and the control group in all previous analyses. The findings show that (1) respondents in the high ESE group were more likely to frame the situation as an opportunity than respondents in the control group or in the low ESE group, (2) when framing situations as an opportunity, individuals are more likely to prefer effectual logic in their entrepreneurial decision-making, and (3) respondents in the high ESE group were more likely to use effectual logic than respondents in the control group or the low ESE group (See Figure 3). Finally, (4) the indirect effects of ESE predicting effectual logic through opportunity framing was significant (high ESE vs. control: $95 \% \mathrm{CI}=$ [0.02, 0.79]; high ESE vs. low ESE: 95\% CI $=[0.01,0.70])$. Overall, the results provide support for the conclusion that novices' higher on ESE, compared with a control group and with the low ESE group, used effectual logic to a larger extent, and that this was as a consequence of them framing an uncertain situation as an opportunity.

INSERT FIGURE 3 ABOUT HERE

\section{Discussion}

Already in the late 1800's William James famously proposed that "If you want a quality, act as if you already had it." Adopting a similar approach, the primary objective of this study was to investigate whether novice entrepreneurs, lacking any entrepreneurial experience, can apply an expert decision-logic solely as a consequence of feeling more confident about their entrepreneurial abilities? At the outset, our findings are consistent with prior research in showing that, on average, novice entrepreneurs use predictive logic in an attempt to foresee future events, even if these events are utterly unpredictable (e.g., Dew et al., 2009). However, we point to the critical role of ESE, regardless of either actual ability or experience, in propelling novices away from their default response and switching to effectual logic. In addition, our results provide support for studies showing how efficacy beliefs influence situational framing (Krueger and Dickson, 1994; Mohammed and Billings, 2002; Wood and Bandura, 1989), and how these positive projections of the self drive entrepreneurial decisions and actions (Dutton and Jackson, 1987; Gartner et al., 2008; Jackson and Dutton, 1988).

We therefore identify our main contribution in showing that effectual logic, which was originally hyped as an expert decision-logic (Sarasvathy, 2008), can also, perhaps more mundanely, stem from an increase in ESE. These findings may begin to resolve an existing tension in the literature, namely; whether effectuation is a distinct feature of expert thinking (Dew et al., 2009a) or if it is merely a constituent of human reasoning more generally (Sarasvathy, 2001). While we do not reject the first, we find support for the later and thereby extend the applicability of effectuation theory to the study of all entrepreneurs, novices and experts alike. As an extension, and granted the caveat that based on our sample we cannot draw strong conclusions about expertise as such, this study may also contribute to research about what it is exactly that expert entrepreneurs become better at (Baron, 2009; Dew et al., 2009a; Sarasvathy, 2008). Given that starting a new venture almost always involves high levels of uncertainty, ESE may well be one of the most important aspects to be learned through 
entrepreneurial experience. We thus contend that overconfidence in entrepreneurial ability, which is routinely portrayed as a cognitive error (Hayward et al., 2006), may also have a positive impact on entrepreneurial decision-making, as long as confidence in prediction is low (e.g., high perceived state uncertainty). In sum....

\section{References}




\section{Tables}

\section{Table 1}

Means, Standard Deviations, and Correlations of Measured Variables.

\begin{tabular}{|c|c|c|c|c|c|c|c|c|c|c|c|c|c|c|c|c|c|}
\hline & \multirow[b]{3}{*}{ Variables } & \multirow{2}{*}{\multicolumn{2}{|c|}{$\begin{array}{c}\text { Low ESE } \\
n=31\end{array}$}} & \multirow{2}{*}{\multicolumn{2}{|c|}{$\begin{array}{l}\text { Control } \\
n=31\end{array}$}} & \multirow{2}{*}{\multicolumn{2}{|c|}{$\begin{array}{c}\text { High ESE } \\
n=31\end{array}$}} & \multirow[b]{3}{*}{1} & \multirow[b]{3}{*}{2} & \multirow[b]{3}{*}{3} & \multirow[b]{3}{*}{4} & \multirow[b]{3}{*}{5} & \multirow[b]{3}{*}{6} & \multirow[b]{3}{*}{7} & \multirow[b]{3}{*}{8} & \multirow[b]{3}{*}{9} & \multirow[b]{3}{*}{10} \\
\hline & & & & & & & & & & & & & & & & & \\
\hline & & $M$ & $S D$ & $M$ & $S D$ & $M$ & $S D$ & & & & & & & & & & \\
\hline 1 & Age & 24.39 & 1.87 & 23.55 & 1.23 & 23.45 & 1.57 & - & & & & & & & & & \\
\hline 2 & Sex $($ male $=1 ;$ female $=2)$ & 1.68 & 0.48 & 1.48 & 0.51 & 1.68 & 0.48 & -0.16 & - & & & & & & & & \\
\hline 3 & Risk Taking Propensity & 1.87 & 1.46 & 2.07 & 1.55 & 2.19 & 1.28 & 0.18 & $-.26 *$ & - & & & & & & & \\
\hline 4 & Meta-Cognitive Knowledge & 3.73 & 0.67 & 3.46 & 0.60 & 3.51 & 0.58 & 0.04 & $.33 * *$ & -0.06 & - & & & & & & \\
\hline 5 & Meta-Cognitive Experience & 3.55 & 0.69 & 3.29 & 0.59 & 3.51 & 0.66 & -0.02 & $.27 * *$ & -0.08 & $.83 * * *$ & - & & & & & \\
\hline 6 & ESE (Baseline) & 2.01 & 0.70 & 1.98 & 0.71 & 2.11 & 0.72 & 0.04 & 0.02 & 0.05 & 0.09 & $.23^{*}$ & - & & & & \\
\hline 7 & ESE (Manipulation check) & 1.90 & 1.06 & 1.89 & 0.81 & 3.54 & 0.61 & -0.02 & -0.03 & 0.15 & 0.02 & 0.14 & $.46 * * *$ & - & & & \\
\hline 8 & Perceived State Uncertainty & 4.16 & 0.76 & 4.15 & 0.84 & 4.15 & 0.53 & 0.18 & -0.11 & $.25^{*}$ & -0.13 & -0.15 & $-.32 * *$ & -0.11 & - & & \\
\hline 9 & Opportunity Framing & 2.60 & 0.91 & 2.50 & 0.53 & 3.74 & 0.87 & -0.09 & 0.15 & 0.03 & 0 & 0.02 & -0.04 & $.58 * * *$ & -0.05 & - & \\
\hline 10 & Effectual Logic & -1.12 & 1.28 & -1.40 & 0.81 & 0.59 & 0.81 & -0.05 & 0.16 & 0.08 & 0.09 & 0.16 & 0.06 & $.63^{* * *}$ & -0.02 & $.54 * * *$ & - \\
\hline
\end{tabular}

Note: Total $\mathrm{n}=93 ; * p<.05 ; * * p<.01 ; * * * p<.001$. 


\section{Figures}

$(\mathrm{H} 2)$

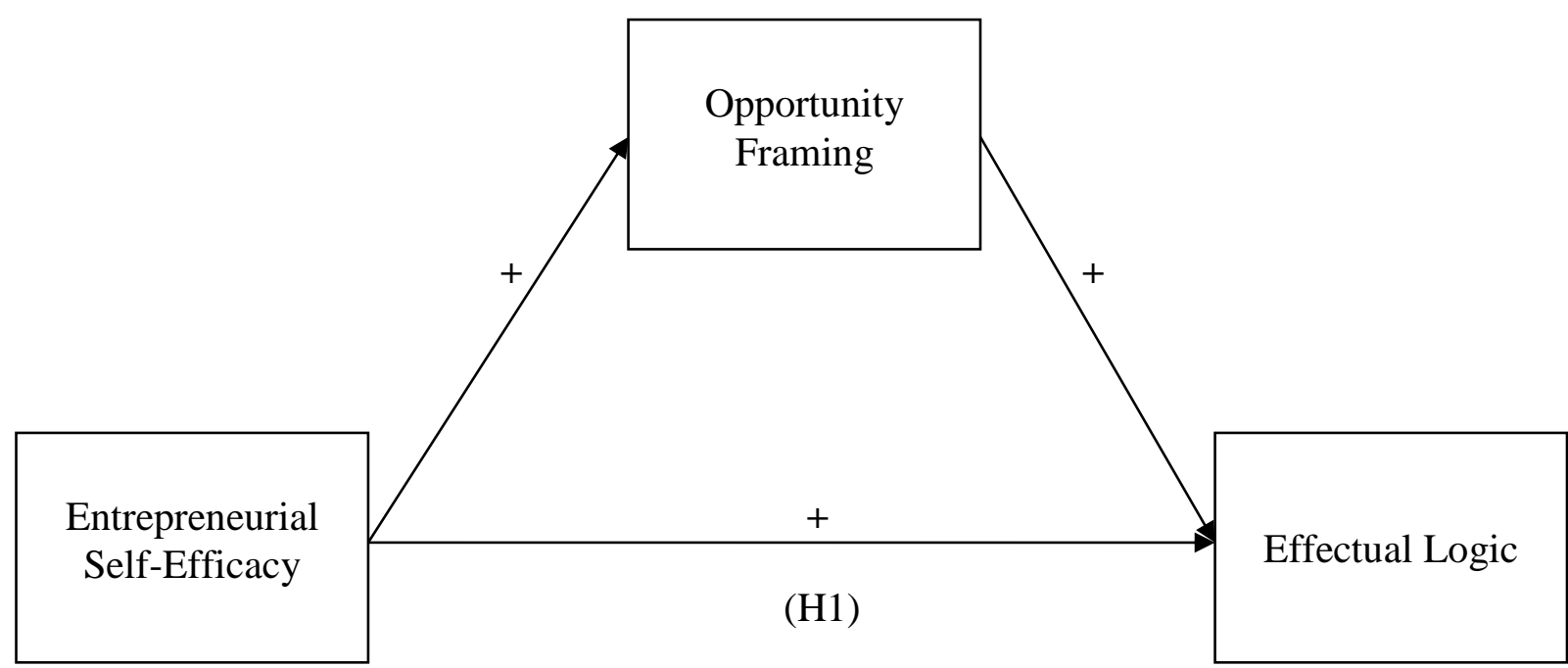

Fig. 1. Conceptual model.

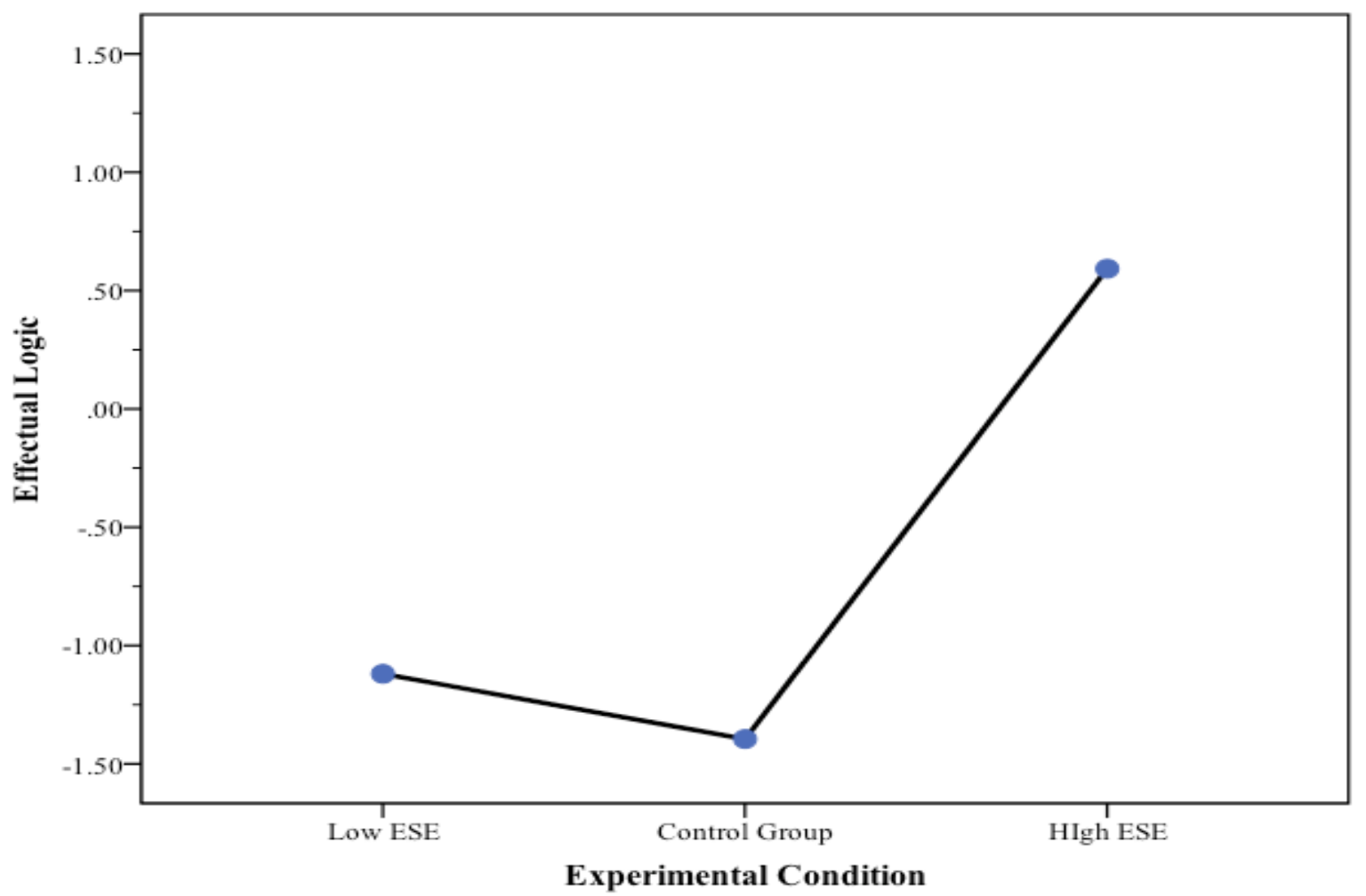

Fig. 2. Main effect of experimental condition on preference for effectual logic 


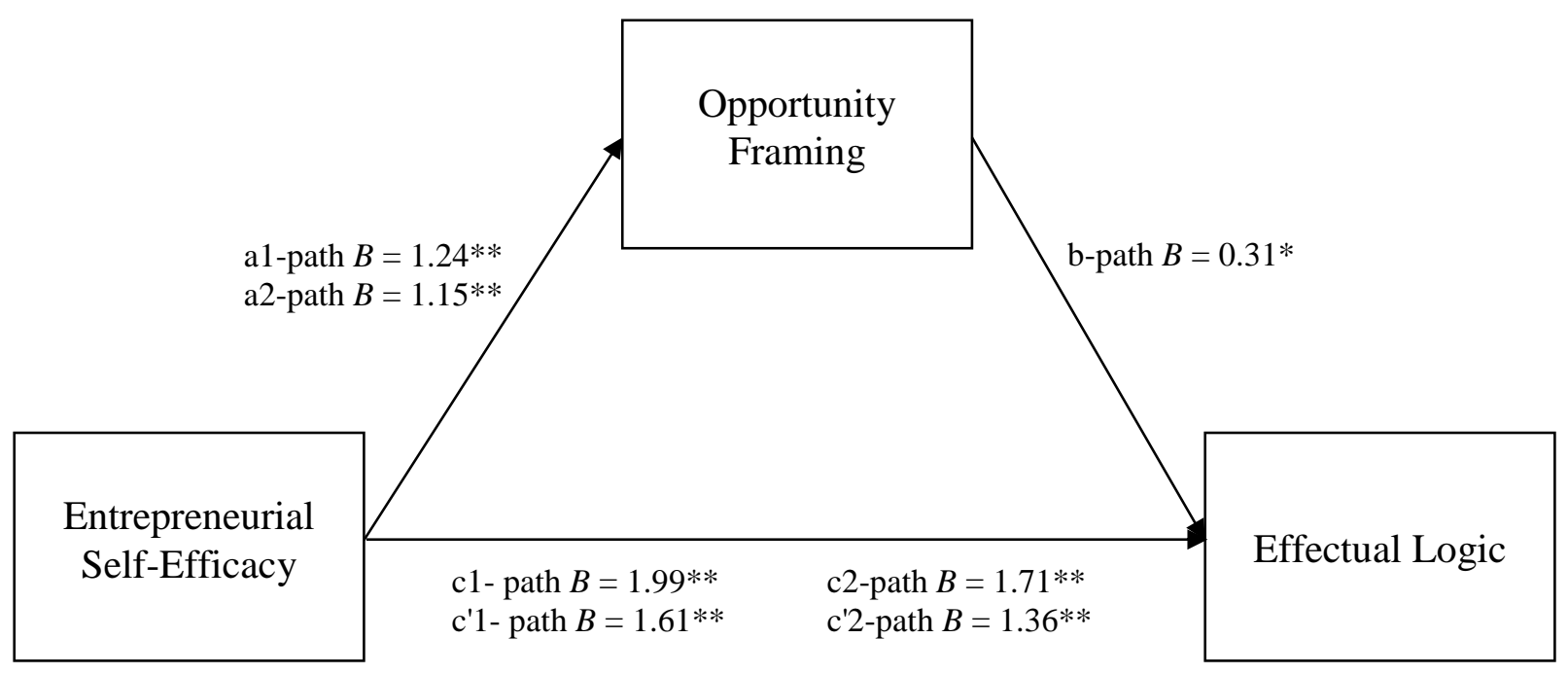

Fig. 3. Mediation model showing the effects of entrepreneurial self-efficacy on effectual logic as mediated by the framing of the situation as an opportunity. Paths marked with the suffix 1 indicate the comparison of High ESE with Control, and paths marked with a suffix 2 indicate the comparison of High ESE with Low ESE (see Hayes \& Preacher, 2013, for a full description of this analytic strategy for testing mediation with a multicategorical predictor). All values are unstandardized coefficients. Asterisks show significant paths $(* p<.05, * * p<.001)$. 